\title{
Octahedral, dicyclic and special linear solutions of some Hamilton-Waterloo problems
}

\author{
Simona Bonvicini \\ Dipartimento di Scienze Fisiche, Informatiche e Matematiche, Università di Modena e \\ Reggio Emilia, Via Campi 213/A, 41125 Modena, Italy \\ Marco Buratti \\ Dipartimento di Matematica e Informatica, Università degli Studi di Perugia, \\ Via Vanvitelli 1, 06123 Perugia, Italy
}

Received 21 April 2016, accepted 23 August 2016, published online 22 March 2017

\begin{abstract}
We give a sharply-vertex-transitive solution of each of the nine Hamilton-Waterloo problems left open by Danziger, Quattrocchi and Stevens.

Keywords: Hamilton-Waterloo problem, group action, octahedral binary group, dicyclic group, special linear group.
\end{abstract}

Math. Subj. Class.: 05C70, 05E18, 05B10

\section{Introduction}

A cycle decomposition of a simple graph $\Gamma=(V, E)$ is a set $\mathcal{D}$ of cycles whose edges partition $E$. A partition $\mathcal{F}$ of $\mathcal{D}$ into classes (2-factors) each of which covers all $V$ exactly once is said to be a 2-factorization of $\Gamma$. The type of a 2 -factor $F$ is the partition $\pi=$ $\left[\ell_{1}^{n_{1}}, \ldots, \ell_{t}^{n_{t}}\right]$ (written in exponential notation) of the integer $|V|$ into the lengths of the cycles of $F$.

A 2-factorization $\mathcal{F}$ of $K_{v}$ (the complete graph of order $v$ ) or $K_{v}-I$ (the cocktail party graph of order $v$ ) whose 2-factors are all of the same type $\pi$ is a solution of the so-called Oberwolfach Problem OP $(v ; \pi)$. If instead the 2-factors of $\mathcal{F}$ are of two different types $\pi$ and $\psi$, then $\mathcal{F}$ is a solution of the so-called Hamilton-Waterloo Problem $\operatorname{HWP}(v ; \pi, \psi ; r, s)$ where $r$ and $s$ denote the number of 2-factors of $\mathcal{F}$ of type $\pi$ and $\psi$, respectively.

A complete solution of the OPs whose 2-factors are uniform, namely of the form $\mathrm{OP}\left(\ell n ;\left[\ell^{n}\right]\right)$, has been given in [1] and [12]. Other important classes of OPs has been

E-mail addresses: simona.bonvicini@unimore.it (Simona Bonvicini), buratti@dmi.unipg.it (Marco Buratti) 
solved in [4, 15]. For the time being, to look for a solution to all possible OPs and, above all, HWPs is too ambitious. Anyway it is reasonable to believe that we are not so far from a complete solution of the HWPs whose 2-factors are uniform, namely of the form $\operatorname{HWP}\left(v ;\left[h^{v / h}\right],\left[w^{v / w}\right] ; r, s\right)$. We can say this especially because of the big progress recently done in [10].

Danziger, Quattrocchi and Stevens [11] treated the HWPs whose 2-factors are either triangle-factors or quadrangle-factors, they namely studied $\operatorname{HWP}\left(12 n ;\left[3^{4 n}\right],\left[4^{3 n}\right] ; r, s\right)$. In the following such an HWP will be denoted, more simply, by $\operatorname{HWP}(12 n ; 3,4 ; r, s)$. They solved this problem for all possible triples $(n, r, s)$ except the following ones:

(i) $(4, r, 23-r)$ with $r \in\{5,7,9,13,15,17\}$;

(ii) $(2, r, 11-r)$ with $r \in\{5,7,9\}$.

Six of the nine above problems have been recently solved in [14] where it was pointed out that all nine problems were also solved in a work still in preparation [2] by the authors of the present paper. Meanwhile, a solution for each of the remaining three problems not considered in [14] have been given in [16]. Notwithstanding, in the present paper we want to present our solutions to the nine HWPs left open by Danziger, Quattrocchi and Stevens in detail. These solutions, differently from those of $[14,16]$, are full of symmetries since they are $G$-regular for a suitable group $G$. We recall that a cycle decomposition (or 2factorization) of a graph $\Gamma$ is said to be $G$-regular when it admits $G$ as an automorphism group acting sharply transitively on all vertices. Here is explicitly our main result:

Theorem 1.1. There exists a $\bar{O}$-regular 2-factorization of $K_{48}-I$ having $r$ trianglefactors and $23-r$ quadrangle-factors where $\bar{O}$ is the binary octahedral group and $r \in$ $\{5,7,9,13,15,17\}$.

There exists a $Q_{24}$-regular 2-factorization of $K_{24}-I$ having $r$ triangle-factors and $11-r$ quadrangle-factors where $Q_{24}$ is the dicyclic group of order 24 and $r \in\{7,9\}$.

There exists a SL $L_{2}(3)$-regular 2-factorization of $K_{24}-I$ having six triangle-factors and five quadrangle-factors where $S L_{2}(3)$ is the 2-dimensional special linear group over $\mathbb{Z}_{3}$.

\section{Some preliminaries}

The use of the classic method of differences allowed to get cyclic (namely $Z_{v}$-regular) solutions of some HWPs in [8, 9, 13]. Now we summarize, in the shortest possible way, the method of partial differences. This method, explained in [7] and successfully applied in many papers (see, especially, [6]), has been also useful for the investigation of $G$-regular 2 -factorizations of a complete graph of odd order [9]. The $G$-regular 2-factorizations of a cocktail party graph can be treated similarly.

Throughout this paper any group $G$ will be assumed to be written multiplicatively and its identity element will be denoted by 1 . Let $\Omega$ be a symmetric subset of a group $G$; this means that $1 \notin \Omega$ and that $\omega \in \Omega$ if and only if $\omega^{-1} \in \Omega$. The Cayley graph on $G$ with connection-set $\Omega$, denoted by $\operatorname{Cay}[G: \Omega]$, is the simple graph whose vertices are the elements of $G$ and whose edges are all 2-subsets of $G$ of the form $\{g, \omega g\}$ with $(g, \omega) \in G \times \Omega$.

Remark 2.1. If $\lambda$ is an involution of a group $G$, then Cay $[G: G \backslash\{1, \lambda\}]$ is isomorphic to $K_{|G|}-I$. So, in the following, such a Cayley graph will be always identified with the cocktail party graph of order $|G|$. 
Let $\operatorname{Cycle}(G)$ be the set of all cycles with vertices in $G$ and consider the natural right action of $G$ on Cycle $(G)$ defined by $\left(c_{1}, c_{2}, \ldots, c_{n}\right)^{g}=\left(c_{1} g, c_{2} g, \ldots, c_{n} g\right)$ for every $C=\left(c_{1}, c_{2}, \ldots, c_{n}\right) \in \operatorname{Cycle}(G)$ and every $g \in G$. The stabilizer and the orbit of any $C \in \operatorname{Cycle}(G)$ under this action will be denoted by $\operatorname{Stab}(C)$ and $\operatorname{Orb}(C)$, respectively. The list of differences of $C \in \operatorname{Cycle}(G)$ is the multiset $\Delta C$ of all possible quotients $x y^{-1}$ with $(x, y)$ an ordered pair of adjacent vertices of $C$. One can see that the multiplicity $m_{\Delta C}(g)$ of any element $g \in G$ in $\Delta C$ is a multiple of the order of $\operatorname{Stab}(C)$. Thus it makes sense to speak of the list of partial differences of $C$ as the multiset $\partial C$ on $G$ in which the multiplicity of any $g \in G$ is defined by

$$
m_{\partial C}(g):=\frac{m_{\Delta C}(g)}{|\operatorname{Stab}(C)|} .
$$

We underline the fact that $\partial C$ is, in general, a multiset. Note that if $\partial C$ is a set, namely without repeated elements, then it is symmetric so that it makes sense to speak of the Cayley graph Cay $[G: \partial C]$. The following elementary but crucial result holds.

Lemma 2.2. If $C \in C y c l e(G)$ and $\partial C$ does not have repeated elements, then $\operatorname{Orb}(C)$ is a $G$-regular cycle-decomposition of $\mathrm{Cay}[G: \partial C]$.

By Remark 2.1, as an immediate consequence of the above lemma we can state the following result.

Theorem 2.3. Let $\lambda$ be an involution of a group $G$. If $\left\{C_{1}, \ldots, C_{t}\right\}$ is a subset of $C y c l e(G)$ such that $\bigcup_{i=1}^{t} \partial C_{i}=G \backslash\{1, \lambda\}$, then $\bigcup_{i=1}^{t} \operatorname{Orb}\left(C_{i}\right)$ is a $G$-regular cycle-decomposition of $K_{|G|}-I$.

We need, as last ingredient, the following easy remarks.

Remark 2.4. If $C \in C y c l e(G)$ and $V(C)$ is a subgroup of $G$, then $\operatorname{Orb}(C)$ is a 2-factor of the complete graph on $G$ whose stabilizer is the whole $G$.

If $C_{1}, \ldots, C_{t}$ are cycles of Cycle $(G)$ and $\bigcup_{i=1}^{t} V\left(C_{i}\right)$ is a complete system of representatives for the left cosets of a subgroup $S$ of $G$, then $\bigcup_{i=1}^{t} O r b_{S}\left(C_{i}\right)$ is a 2-factor of the complete graph on $G$ whose stabilizer is $S$.

\section{Octahedral solutions of six Hamilton-Waterloo problems}

Throughout this section $G$ will denote the so-called binary octahedral group which is usually denoted by $\bar{O}$. This group, up to isomorphism, can be viewed as a group of units of the skew-field $\mathbb{H}$ of quaternions introduced by Hamilton, that is an extension of the complex field $\mathbb{C}$. We recall the basic facts regarding $\mathbb{H}$. Its elements are all real linear combinations of $1, i, j$ and $k$. The sum and the product of two quaternions are defined in the natural way under the rules that

$$
i^{2}=j^{2}=k^{2}=i j k=-1 .
$$

If $q=a+b i+c j+d k \neq 0$, then the inverse of $q$ is given by

$$
q^{-1}=\frac{a-b i-c j-d k}{a^{2}+b^{2}+c^{2}+d^{2}} .
$$


The 48 elements of the multiplicative group $G$ are the following:

$$
\begin{aligned}
& \pm 1, \pm i, \pm j, \pm k \\
& \frac{1}{2}( \pm 1 \pm i \pm j \pm k) \\
& \frac{1}{\sqrt{2}}( \pm x \pm y), \quad\{x, y\} \in\left(\frac{\{1, i, j, k\}}{2}\right) .
\end{aligned}
$$

The use of the octahedral group $G$ was crucial in [3] to get a Steiner triple system of any order $v=96 n+49$ with an automorphism group acting sharply transitively an all but one point. Here $G$ will be used to get a $G$-regular solution of each of the six Hamilton-Waterloo problems of order 48 left open in [11]. We will need to consider the following subgroups of $G$ of order 16 and 12 , respectively:

- $K=\left\langle k, \frac{1}{\sqrt{2}}(j-k)\right\rangle$;

- $L=\left\langle\frac{1}{\sqrt{2}}(j-k), \frac{1}{2}(-1-i+j+k)\right\rangle$.

\subsection{An octahedral solution of $\operatorname{HWP}(48 ; 3,4 ; 5,18)$}

Consider the nine cycles of $\operatorname{Cycle}(G)$ defined as follows.

$$
\begin{aligned}
& C_{1}=\left(1,-\frac{1}{\sqrt{2}}(1-k), \frac{1}{2}(1-i-j-k)\right) \\
& C_{2}=\left(1, \frac{1}{2}(-1-i+j+k), \frac{1}{2}(-1+i-j-k)\right) \\
& C_{3}=\left(1, \frac{1}{2}(-1+i+j-k), \frac{1}{2}(-1-i-j+k)\right) \\
& C_{4}=(1, k,-1,-k) \\
& C_{5}=(1, j,-1,-j) \\
& C_{6}=\left(1, \frac{1}{\sqrt{2}}(-i+k),-\frac{1}{2}(1+i+j+k),-\frac{1}{\sqrt{2}}(j+k)\right) \\
& C_{7}=\left(1, \frac{1}{\sqrt{2}}(i-j), \frac{1}{\sqrt{2}}(1+i), \frac{1}{2}(1-i-j+k)\right) \\
& C_{8}=\left(1, \frac{1}{2}(1-i+j-k), k,-\frac{1}{\sqrt{2}}(1+j)\right) \\
& C_{9}=\left(1, \frac{1}{\sqrt{2}}(1-i),-\frac{1}{\sqrt{2}}(1+i), \frac{1}{2}(-1-i+j-k)\right)
\end{aligned}
$$

We note that $\operatorname{Stab}\left(C_{i}\right)=V\left(C_{i}\right)$ for $2 \leq i \leq 5$ while all other $C_{i}$ 's have trivial stabilizer. Thus, by Lemma 2.2, one can check that $\operatorname{Orb}\left(C_{i}\right)$ is a $\ell_{i}$-cycle decomposition of Cay $\left[G: \Omega_{i}\right]$ where $\ell_{i}$ is the length of $C_{i}$ and where the $\Omega_{i}$ 's are the symmetric subsets of $G$ listed below.

$$
\begin{aligned}
& \Omega_{1}=\left\{-\frac{1}{\sqrt{2}}(1-k), \frac{1}{2}(1-i-j-k),-\frac{1}{\sqrt{2}}(1+i)\right\}^{ \pm 1} \\
& \Omega_{2}=\left\{\frac{1}{2}(-1-i+j+k)\right\}^{ \pm 1} \\
& \Omega_{3}=\left\{\frac{1}{2}(-1+i+j-k)\right\}^{ \pm 1} \\
& \Omega_{4}=\{k\}^{ \pm 1} \\
& \Omega_{5}=\{j\}^{ \pm 1} \\
& \Omega_{6}=\left\{\frac{1}{\sqrt{2}}(-i+k), \frac{1}{\sqrt{2}}(j-k), \frac{1}{\sqrt{2}}(1-k),-\frac{1}{\sqrt{2}}(j+k)\right\}^{ \pm 1}
\end{aligned}
$$




$$
\begin{aligned}
& \Omega_{7}=\left\{\frac{1}{\sqrt{2}}(i-j), \frac{1}{2}(1+i-j-k), \frac{1}{\sqrt{2}}(i+j), \frac{1}{2}(1-i-j+k)\right\}^{ \pm 1} \\
& \Omega_{8}=\left\{\frac{1}{2}(1-i+j-k),-\frac{1}{2}(1+i+j+k),-\frac{1}{\sqrt{2}}(i+k),-\frac{1}{\sqrt{2}}(1+j)\right\}^{ \pm 1} \\
& \Omega_{9}=\left\{\frac{1}{\sqrt{2}}(1-i), i, \frac{1}{\sqrt{2}}(1+j), \frac{1}{2}(-1-i+j-k)\right\}^{ \pm 1}
\end{aligned}
$$

One can see that the $\Omega_{i}$ 's partition $G \backslash\{1,-1\}$. Thus, by Theorem 2.3 we can say that $\mathcal{C}:=\bigcup_{i=1}^{9} \operatorname{Orb}_{G}\left(C_{i}\right)$ is a $G$-regular cycle-decomposition of $K_{48}-I$. Now set $F_{i}=$ $\operatorname{Orb}_{S_{i}}\left(C_{i}\right)$ where

$$
S_{i}= \begin{cases}K & \text { for } i=1 \\ G & \text { for } 2 \leq i \leq 5 \\ L & \text { for } 6 \leq i \leq 9\end{cases}
$$

By Remark 2.4, each $F_{i}$ is a 2-factor of $K_{48}-I$ with $\operatorname{Stab}\left(F_{i}\right)=S_{i}$, hence $\operatorname{Orb}\left(F_{i}\right)$ has length 3 or 1 or 4 according to whether $i=1$, or $2 \leq i \leq 5$, or $6 \leq i \leq 9$, respectively. The cycles of $F_{i}$ are triangles or quadrangles according to whether or not $i \leq 3$. Thus, recalling that $\mathcal{C}$ is a cycle-decomposition of $K_{48}-I$, we conclude that $\mathcal{F}:=\bigcup_{i=1}^{9} \operatorname{Orb}\left(F_{i}\right)$ is a $G$-regular 2-factorization of $K_{48}-I$ with 5 triangle-factors and 18 quadrangle-factors, namely a $G$-regular solution of $\operatorname{HWP}(48 ; 3,4 ; 5,18)$.

\subsection{An octahedral solution of $\operatorname{HWP}(48 ; 3,4 ; 7,16)$}

Consider the seven cycles of $\operatorname{Cycle}(G)$ defined as follows.

$$
\begin{aligned}
& C_{1}=\left(1,-\frac{1}{\sqrt{2}}(i+j), \frac{1}{2}(1-i+j+k)\right) \\
& C_{2}=\left(1, \frac{1}{2}(-1-i+j+k), \frac{1}{2}(1-i-j-k)\right) \\
& C_{3}=\left(1, \frac{1}{2}(-1+i+j-k), \frac{1}{2}(-1-i-j+k)\right) \\
& C_{4}=\left(1, \frac{1}{\sqrt{2}}(-i+k), \frac{1}{2}(1+i+j-k),-\frac{1}{\sqrt{2}}(j+k)\right) \\
& C_{5}=\left(1, \frac{1}{\sqrt{2}}(i-j), \frac{1}{\sqrt{2}}(1-k), \frac{1}{\sqrt{2}}(1+i)\right) \\
& C_{6}=\left(1, \frac{1}{\sqrt{2}}(1+k),-\frac{1}{2}(1+i+j+k), \frac{1}{\sqrt{2}}(1+j)\right) \\
& C_{7}=\left(1,-\frac{1}{2}(1+i+j+k), \frac{1}{2}(1-i+j-k), \frac{1}{2}(1-i-j+k)\right)
\end{aligned}
$$

We note that $\operatorname{Stab}\left(C_{3}\right)=V\left(C_{3}\right)$ while all other $C_{i}$ 's have trivial stabilizer. Thus, by Lemma 2.2, one can check that $\operatorname{Orb}\left(C_{i}\right)$ is a $\ell_{i}$-cycle decomposition of Cay $\left[G: \Omega_{i}\right]$ where $\ell_{i}$ is the length of $C_{i}$ and where the $\Omega_{i}$ 's are the symmetric subsets of $G$ listed below.

$$
\begin{aligned}
& \Omega_{1}=\left\{-\frac{1}{\sqrt{2}}(i+j), \frac{1}{2}(1-i+j+k), \frac{1}{\sqrt{2}}(-j+k)\right\}^{ \pm 1} \\
& \Omega_{2}=\left\{\frac{1}{2}(-1-i+j+k), \frac{1}{2}(1-i-j-k), \frac{1}{2}(-1-i+j-k)\right\}^{ \pm 1} \\
& \Omega_{3}=\left\{\frac{1}{2}(-1+i+j-k)\right\}^{ \pm 1} \\
& \Omega_{4}=\left\{\frac{1}{\sqrt{2}}(-i+k),-\frac{1}{\sqrt{2}}(1-k), \frac{1}{\sqrt{2}}(i+k),-\frac{1}{\sqrt{2}}(j+k)\right\}^{ \pm 1} \\
& \Omega_{5}=\left\{\frac{1}{\sqrt{2}}(i-j),-j, \frac{1}{2}(1-i+j-k), \frac{1}{\sqrt{2}}(1+i)\right\}^{ \pm 1} \\
& \Omega_{6}=\left\{\frac{1}{\sqrt{2}}(1+k), \frac{1}{\sqrt{2}}(-1+j),-\frac{1}{\sqrt{2}}(1+i), \frac{1}{\sqrt{2}}(1+j)\right\}^{ \pm 1} \\
& \Omega_{7}=\left\{-\frac{1}{2}(1+i+j+k),-i,-k, \frac{1}{2}(1-i-j+k)\right\}^{ \pm 1}
\end{aligned}
$$


One can see that the $\Omega_{i}$ 's partition $G \backslash\{1,-1\}$. Thus, by Theorem 2.3 we can say that $\mathcal{C}:=\bigcup_{i=1}^{7} \operatorname{Orb}_{G}\left(C_{i}\right)$ is a $G$-regular cycle-decomposition of $K_{48}-I$. Now set $F_{i}=$ $\operatorname{Orb}_{S_{i}}\left(C_{i}\right)$ where

$$
S_{i}= \begin{cases}K & \text { for } i=1,2 \\ G & \text { for } i=3 \\ L & \text { for } 4 \leq i \leq 7\end{cases}
$$

By Remark 2.4, each $F_{i}$ is a 2-factor of $K_{48}-I$ with $\operatorname{Stab}_{G}\left(F_{i}\right)=S_{i}$, hence $\operatorname{Orb}_{G}\left(F_{i}\right)$ has length 3 or 1 or 4 according to whether $i=1,2$ or $i=3$ or $4 \leq i \leq 7$, respectively.

The cycles of $F_{i}$ are triangles or quadrangles according to whether or not $i \leq 3$. Thus, recalling that $\mathcal{C}$ is a cycle-decomposition of $K_{48}-I$, we conclude that $\mathcal{F}:=$ $\bigcup_{i=1}^{7} \operatorname{Orb}_{G}\left(F_{i}\right)$ is a $G$-regular 2-factorization of $K_{48}-I$ with 7 triangle-factors and 16 quadrangle-factors, namely a $G$-regular solution of $\operatorname{HWP}(48 ; 3,4 ; 7,16)$.

\subsection{An octahedral solution of $\operatorname{HWP}(48 ; 3,4 ; 9,14)$}

Consider the eight cycles of $\operatorname{Cycle}(G)$ defined as follows.

$$
\begin{aligned}
& C_{1}=\left(1, \frac{1}{\sqrt{2}}(i+j), \frac{1}{2}(1-i-j-k)\right) \\
& C_{2}=\left(1,-\frac{1}{\sqrt{2}}(1-k), \frac{1}{\sqrt{2}}(1+j)\right) \\
& C_{3}=\left(1, \frac{1}{2}(-1-i+j+k), \frac{1}{2}(1+i-j+k)\right) \\
& C_{4}=\left(1, \frac{1}{\sqrt{2}}(-i+k), \frac{1}{\sqrt{2}}(1-i), \frac{1}{2}(-1-i+j-k)\right) \\
& C_{5}=\left(1, \frac{1}{\sqrt{2}}(i-j), \frac{1}{2}(-1+i+j+k),-\frac{1}{\sqrt{2}}(j+k)\right) \\
& C_{6}=\left(1, \frac{1}{\sqrt{2}}(1+i), \frac{1}{\sqrt{2}}(1-i), \frac{1}{2}(1-i-j+k)\right) \\
& C_{7}=(1, k,-1,-k) \\
& C_{8}=(1, j,-1,-j)
\end{aligned}
$$

We note that $\operatorname{Stab}\left(C_{i}\right)=V\left(C_{i}\right)$ for $i=7,8$ while all other $C_{i}$ 's have trivial stabilizer. By Lemma 2.2, one can check that $\operatorname{Orb}\left(C_{i}\right)$ is a $\ell_{i}$-cycle decomposition of Cay $\left[G: \Omega_{i}\right]$ where $\ell_{i}$ is the length of $C_{i}$ and where the $\Omega_{i}$ 's are the symmetric subsets of $G$ listed below.

$$
\begin{aligned}
& \Omega_{1}=\left\{\frac{1}{\sqrt{2}}(i+j), \frac{1}{2}(1-i-j-k), \frac{1}{\sqrt{2}}(-1+i)\right\}^{ \pm 1} \\
& \Omega_{2}=\left\{-\frac{1}{\sqrt{2}}(1-k), \frac{1}{\sqrt{2}}(1+j), \frac{1}{2}(-1+i+j+k)\right\}^{ \pm 1} \\
& \Omega_{3}=\left\{\frac{1}{2}(-1-i+j+k), \frac{1}{2}(1+i-j+k), \frac{1}{2}(-1-i-j+k)\right\}^{ \pm 1} \\
& \Omega_{4}=\left\{\frac{1}{\sqrt{2}}(-i+k), \frac{1}{2}(1-i+j+k), \frac{1}{\sqrt{2}}(i+k), \frac{1}{2}(-1-i+j-k)\right\}^{ \pm 1} \\
& \Omega_{5}=\left\{\frac{1}{\sqrt{2}}(i-j), \frac{1}{\sqrt{2}}(j-k),-\frac{1}{\sqrt{2}}(1+j),-\frac{1}{\sqrt{2}}(j+k)\right\}^{ \pm 1} \\
& \Omega_{6}=\left\{\frac{1}{\sqrt{2}}(1+i), i, \frac{1}{\sqrt{2}}(1-k), \frac{1}{2}(1-i-j+k)\right\}^{ \pm 1} \\
& \Omega_{7}=\{k\}^{ \pm 1} \\
& \Omega_{8}=\{j\}^{ \pm 1}
\end{aligned}
$$

Now note that the $\Omega_{i}$ 's partition $G \backslash\{1,-1\}$. Thus, by Theorem 2.3 we can say that $\mathcal{C}:=$ $\bigcup_{i=1}^{8} \operatorname{Orb}\left(C_{i}\right)$ is a $G$-regular cycle-decomposition of $K_{48}-I$. Now set $F_{i}=\operatorname{Orb}_{S_{i}}\left(C_{i}\right)$ 
where

$$
S_{i}= \begin{cases}K & \text { for } 1 \leq i \leq 3 \\ L & \text { for } 4 \leq i \leq 6 \\ G & \text { for } i=7,8\end{cases}
$$

By Remark 2.4, each $F_{i}$ is a 2-factor of $K_{48}-I$ with $\operatorname{Stab}_{G}\left(F_{i}\right)=S_{i}$, hence $\operatorname{Orb}_{G}\left(F_{i}\right)$ has length 3 or 4 or 1 according to whether $1 \leq i \leq 3$ or $4 \leq i \leq 6$ or $i=7,8$, respectively. The cycles of $F_{i}$ are triangles or quadrangles according to whether or not $i \leq 3$. Thus, recalling that $\mathcal{C}$ is a cycle-decomposition of $K_{48}-I$, we conclude that $\mathcal{F}:=\bigcup_{i=1}^{8} \operatorname{Orb}_{G}\left(F_{i}\right)$ is a $G$-regular 2-factorization of $K_{48}-I$ with 9 triangle-factors and 14 quadrangle-factors, namely a $G$-regular solution of $\operatorname{HWP}(48 ; 3,4 ; 9,14)$.

\subsection{An octahedral solution of $\operatorname{HWP}(48 ; 3,4 ; 13,10)$}

Consider the nine cycles of $\operatorname{Cycle}(G)$ defined as follows.

$$
\begin{aligned}
& C_{1}=\left(1,-\frac{1}{\sqrt{2}}(i+j),-\frac{1}{\sqrt{2}}(1+j)\right) \\
& C_{2}=\left(1, \frac{1}{2}(1-i+j-k),-\frac{1}{\sqrt{2}}(i+k)\right) \\
& C_{3}=\left(1, \frac{1}{\sqrt{2}}(-i+j), \frac{1}{2}(1-i-j-k)\right) \\
& C_{4}=\left(1, \frac{1}{2}(-1+i-j+k), \frac{1}{\sqrt{2}}(i-k)\right) \\
& C_{5}=\left(1, \frac{1}{2}(-1-i+j+k), \frac{1}{2}(-1+i-j-k)\right) \\
& C_{6}=(1, k,-1,-k) \\
& C_{7}=(1, j,-1,-j) \\
& C_{8}=\left(1,-\frac{1}{2}(1+i+j+k), \frac{1}{2}(-1+i-j+k), \frac{1}{\sqrt{2}}(1+j)\right) \\
& C_{9}=\left(1,-\frac{1}{\sqrt{2}}(1+k),-k, \frac{1}{2}(-1+i+j-k)\right)
\end{aligned}
$$

We note that $\operatorname{Stab}\left(C_{i}\right)=V\left(C_{i}\right)$ for $5 \leq i \leq 7$ while all other $C_{i}$ 's have trivial $G$ stabilizer. Thus, by Lemma 2.2, one can check that $\operatorname{Orb}\left(C_{i}\right)$ is a $\ell_{i}$-cycle decomposition of Cay $\left[G: \Omega_{i}\right]$ where $\ell_{i}$ is the length of $C_{i}$ and where the $\Omega_{i}$ 's are the symmetric subsets of $G$ listed below.

$$
\begin{aligned}
& \Omega_{1}=\left\{-\frac{1}{\sqrt{2}}(i+j),-\frac{1}{\sqrt{2}}(1+j), \frac{1}{2}(1+i+j-k)\right\}^{ \pm 1} \\
& \Omega_{2}=\left\{\frac{1}{2}(1-i+j-k),-\frac{1}{\sqrt{2}}(i+k), \frac{1}{\sqrt{2}}(1+i)\right\}^{ \pm 1} \\
& \Omega_{3}=\left\{\frac{1}{\sqrt{2}}(-i+j), \frac{1}{2}(1-i-j-k), \frac{1}{\sqrt{2}}(j-k)\right\}^{ \pm 1} \\
& \Omega_{4}=\left\{\frac{1}{2}(-1+i-j+k), \frac{1}{\sqrt{2}}(i-k),-\frac{1}{\sqrt{2}}(j+k)\right\}^{ \pm 1} \\
& \Omega_{5}=\left\{\frac{1}{2}(-1-i+j+k)\right\}^{ \pm 1} \\
& \Omega_{6}=\{k\}^{ \pm 1} \\
& \Omega_{7}=\{j\}^{ \pm 1} \\
& \Omega_{8}=\left\{-\frac{1}{2}(1+i+j+k), i, \frac{1}{\sqrt{2}}(-1+i), \frac{1}{\sqrt{2}}(1+j)\right\}^{ \pm 1} \\
& \Omega_{9}=\left\{-\frac{1}{\sqrt{2}}(1+k), \frac{1}{\sqrt{2}}(1-k), \frac{1}{2}(1-i+j+k), \frac{1}{2}(-1+i+j-k)\right\}^{ \pm 1}
\end{aligned}
$$


Now note that the $\Omega_{i}$ 's partition $G \backslash\{1,-1\}$. Thus, by Theorem 2.3 we can say that $\mathcal{C}:=$ $\bigcup_{i=1}^{9} \operatorname{Orb}\left(C_{i}\right)$ is a $G$-regular cycle-decomposition of $K_{48}-I$. Now set $F_{i}=\operatorname{Orb}_{S_{i}}\left(C_{i}\right)$ where

$$
S_{i}= \begin{cases}K & \text { for } 1 \leq i \leq 4 \\ G & \text { for } 5 \leq i \leq 7 \\ L & \text { for } i=8,9\end{cases}
$$

By Remark 2.4, each $F_{i}$ is a 2-factor of $K_{48}-I$ with $\operatorname{Stab}_{G}\left(F_{i}\right)=S_{i}$, hence $\operatorname{Orb}_{G}\left(F_{i}\right)$ has length 3 or 1 or 4 according to whether $1 \leq i \leq 4$ or $5 \leq i \leq 7$ or $i=8,9$, respectively.

The cycles of $F_{i}$ are triangles or quadrangles according to whether or not $i \leq 5$. Thus, recalling that $\mathcal{C}$ is a cycle-decomposition of $K_{48}-I$, we conclude that $\mathcal{F}:=$ $\bigcup_{i=1}^{9} \operatorname{Orb}_{G}\left(F_{i}\right)$ is a $G$-regular 2-factorization of $K_{48}-I$ with 13 triangle-factors and 10 quadrangle-factors, namely a $G$-regular solution of $\operatorname{HWP}(48 ; 3,4 ; 13,10)$.

\subsection{An octahedral solution of $\operatorname{HWP}(48 ; 3,4 ; 15,8)$}

Consider the seven cycles of $\operatorname{Cycle}(G)$ defined as follows.

$$
\begin{aligned}
& C_{1}=\left(1, \frac{1}{2}(-1-i+j+k), \frac{1}{\sqrt{2}}(i+k)\right) \\
& C_{2}=\left(1,-\frac{1}{\sqrt{2}}(i+j),-\frac{1}{\sqrt{2}}(1+j)\right) \\
& C_{3}=\left(1, \frac{1}{2}(-1+i+j-k), \frac{1}{2}(1-i+j+k)\right) \\
& C_{4}=\left(1, \frac{1}{2}(1+i+j+k), \frac{1}{\sqrt{2}}(1+j)\right) \\
& C_{5}=\left(1, \frac{1}{2}(1-i+j-k), \frac{1}{\sqrt{2}}(i-k)\right) \\
& C_{6}=\left(1,-j, k,-\frac{1}{\sqrt{2}}(1-k)\right) \\
& C_{7}=\left(1, \frac{1}{\sqrt{2}}(i-j), \frac{1}{2}(-1-i+j-k), \frac{1}{2}(-1+i+j+k)\right)
\end{aligned}
$$

Here, every $C_{i}$ has trivial stabilizer. Thus, by Lemma 2.2, one can check that $\operatorname{Orb}\left(C_{i}\right)$ is a $\ell_{i}$-cycle decomposition of $\operatorname{Cay}\left[G: \Omega_{i}\right]$ where $\ell_{i}$ is the length of $C_{i}$ and where the $\Omega_{i}$ 's are the symmetric subsets of $G$ listed below.

$$
\begin{aligned}
& \Omega_{1}=\left\{\frac{1}{2}(-1-i+j+k), \frac{1}{\sqrt{2}}(i+k), \frac{1}{\sqrt{2}}(-j+k)\right\}^{ \pm 1} \\
& \Omega_{2}=\left\{-\frac{1}{\sqrt{2}}(i+j),-\frac{1}{\sqrt{2}}(1+j), \frac{1}{2}(1+i+j-k)\right\}^{ \pm 1} \\
& \Omega_{3}=\left\{\frac{1}{2}(-1+i+j-k), \frac{1}{2}(1-i+j+k), \frac{1}{2}(-1-i+j-k)\right\}^{ \pm 1} \\
& \Omega_{4}=\left\{\frac{1}{2}(1+i+j+k), \frac{1}{\sqrt{2}}(1+j), \frac{1}{\sqrt{2}}(1+i)\right\}^{ \pm 1} \\
& \Omega_{5}=\left\{\frac{1}{2}(1-i+j-k), \frac{1}{\sqrt{2}}(i-k), \frac{1}{\sqrt{2}}(j+k)\right\}^{ \pm 1} \\
& \Omega_{6}=\left\{-j,+i, \frac{1}{\sqrt{2}}(1-k),-\frac{1}{\sqrt{2}}(1-k)\right\}^{ \pm 1} \\
& \Omega_{7}=\left\{\frac{1}{\sqrt{2}}(i-j),-\frac{1}{\sqrt{2}}(1+i),+k, \frac{1}{2}(-1+i+j+k)\right\}^{ \pm 1}
\end{aligned}
$$

Now note that the $\Omega_{i}$ 's partition $G \backslash\{1,-1\}$. Thus, by Theorem 2.3 we can say that $\mathcal{C}:=\bigcup_{i=1}^{7} \operatorname{Orb}\left(C_{i}\right)$ is a $G$-regular cycle-decomposition of $K_{48}-I$. Set $F_{i}=\operatorname{Orb}_{S_{i}}\left(C_{i}\right)$ where

$$
S_{i}= \begin{cases}K & \text { for } 1 \leq i \leq 5 \\ L & \text { for } i=6,7\end{cases}
$$


By Remark 2.4, each $F_{i}$ is a 2-factor of $K_{48}-I$ with $\operatorname{Stab}_{G}\left(F_{i}\right)=S_{i}$, hence $\operatorname{Orb}_{G}\left(F_{i}\right)$ has length 3 or 4 according to whether $1 \leq i \leq 5$ or $i=6,7$, respectively. The cycles of $F_{i}$ are triangles or quadrangles according to whether or not $i \leq 5$. Thus, recalling that $\mathcal{C}$ is a cycle-decomposition of $K_{48}-I$, we conclude that $\mathcal{F}:=\bigcup_{i=1}^{\overline{7}} \operatorname{Orb}_{G}\left(F_{i}\right)$ is a $G$-regular 2-factorization of $K_{48}-I$ with 15 triangle-factors and 8 quadrangle-factors, namely a $G$-regular solution of $\operatorname{HWP}(48 ; 3,4 ; 15,8)$.

\subsection{An octahedral solution of $\operatorname{HWP}(48 ; 3,4 ; 17,6)$}

Consider the ten cycles of $\operatorname{Cycle}(G)$ defined as follows.

$$
\begin{aligned}
C_{1} & =\left(1,-\frac{1}{\sqrt{2}}(1-k),-\frac{1}{\sqrt{2}}(i+k)\right) \\
C_{2} & =\left(1,-\frac{1}{\sqrt{2}}(i+j), \frac{1}{2}(-1+i+j+k)\right) \\
C_{3} & =\left(1, \frac{1}{2}(1+i-j-k),-\frac{1}{\sqrt{2}}(1+j)\right) \\
C_{4} & =\left(1, \frac{1}{\sqrt{2}}(-i+j), \frac{1}{\sqrt{2}}(-i+k)\right) \\
C_{5} & =\left(1, \frac{1}{2}(1-i+j-k), \frac{1}{\sqrt{2}}(1-j)\right) \\
C_{6} & =\left(1, \frac{1}{2}(-1-i+j+k), \frac{1}{2}(-1+i-j-k)\right) \\
C_{7} & =\left(1, \frac{1}{2}(-1+i+j-k), \frac{1}{2}(-1-i-j+k)\right) \\
C_{8} & =(1, k,-1,-k) \\
C_{9} & =(1, j,-1,-j) \\
C_{10} & =\left(1, \frac{1}{\sqrt{2}}(1+i), \frac{1}{\sqrt{2}}(1-i), \frac{1}{2}(1-i-j+k)\right)
\end{aligned}
$$

We note that $\operatorname{Stab}\left(C_{i}\right)=V\left(C_{i}\right)$ for $6 \leq i \leq 9$ while all other $C_{i}$ 's have trivial stabilizer. Thus, by Lemma 2.2, one can check that $\operatorname{Orb}\left(C_{i}\right)$ is a $\ell_{i}$-cycle decomposition of Cay $\left[G: \Omega_{i}\right]$ where $\ell_{i}$ is the length of $C_{i}$ and where the $\Omega_{i}$ 's are the symmetric subsets of $G$ listed below.

$$
\begin{aligned}
\Omega_{1} & =\left\{-\frac{1}{\sqrt{2}}(1-k),-\frac{1}{\sqrt{2}}(i+k), \frac{1}{2}(-1-i+j-k)\right\}^{ \pm 1} \\
\Omega_{2} & =\left\{-\frac{1}{\sqrt{2}}(i+j), \frac{1}{2}(-1+i+j+k), \frac{1}{\sqrt{2}}(-1+i)\right\}^{ \pm 1} \\
\Omega_{3} & =\left\{\frac{1}{2}(1+i-j-k),-\frac{1}{\sqrt{2}}(1+j), \frac{1}{\sqrt{2}}(j+k)\right\}^{ \pm 1} \\
\Omega_{4} & =\left\{\frac{1}{\sqrt{2}}(-i+j), \frac{1}{\sqrt{2}}(-i+k), \frac{1}{2}(1-i-j-k)\right\}^{ \pm 1} \\
\Omega_{5} & =\left\{\frac{1}{2}(1-i+j-k), \frac{1}{\sqrt{2}}(1-j), \frac{1}{\sqrt{2}}(j-k)\right\}^{ \pm 1} \\
\Omega_{6} & =\left\{\frac{1}{2}(-1-i+j+k)\right\}^{ \pm 1} \\
\Omega_{7} & =\left\{\frac{1}{2}(-1+i+j-k)\right\}^{ \pm 1} \\
\Omega_{8} & =\{k\}^{ \pm 1} \\
\Omega_{9} & =\{j\}^{ \pm 1} \\
\Omega_{10} & =\left\{\frac{1}{\sqrt{2}}(1+i), i, \frac{1}{\sqrt{2}}(1-k), \frac{1}{2}(1-i-j+k)\right\}^{ \pm 1}
\end{aligned}
$$

Now note that the $\Omega_{i}$ 's partition $G \backslash\{1,-1\}$. Thus, by Lemma 2.2 we can say that $\mathcal{C}:=\bigcup_{i=1}^{10} \operatorname{Orb}\left(C_{i}\right)$ is a $G$-regular cycle-decomposition of $K_{48}-I$. Set $F_{i}=\operatorname{Orb}_{S_{i}}\left(C_{i}\right)$ 
where

$$
S_{i}= \begin{cases}K & \text { for } 1 \leq i \leq 5 \\ G & \text { for } 6 \leq i \leq 9 \\ L & \text { for } i=10\end{cases}
$$

By Remark 2.4, each $F_{i}$ is a 2-factor of $K_{48}$ with $\operatorname{Stab}_{G}\left(F_{i}\right)=S_{i}$, hence $\operatorname{Orb}_{G}\left(F_{i}\right)$ has length 3 or 1 or 4 according to whether $1 \leq i \leq 5$ or $6 \leq i \leq 9$ or $i=10$, respectively. The cycles of $F_{i}$ are triangles or quadrangles according to whether or not $i \leq 7$. Thus, recalling that $\mathcal{C}$ is a cycle-decomposition of $K_{48}-I$, we conclude that $\mathcal{F}:=\bigcup_{i=1}^{10} \operatorname{Orb}_{G}\left(F_{i}\right)$ is a $G$-regular 2-factorization of $K_{48}-I$ with 17 triangle-factors and 6 quadrangle-factors, namely a $G$-regular solution of $\operatorname{HWP}(48 ; 3,4 ; 17,6)$.

\section{Dicyclic solutions of two Hamilton-Waterloo problems}

In this section $G$ will denote the dicyclic group of order 24 which is usually denoted by $Q_{24}$. Thus $G$ has the following presentation:

$$
G=\left\langle a, b \mid a^{12}=1, b^{2}=a^{6}, b^{-1} a b=a^{-1}\right\rangle
$$

Note that the elements of $G$ can be written in the form $a^{i} b^{j}$ with $0 \leq i \leq 11$ and $j=0,1$. The group $G$ has a unique involution which is $a^{6}$ and we will need to consider the following subgroups of $G$ :

- $H=\langle b\rangle=\left\{1, b, a^{6}, a^{6} b\right\}$;

- $K=\left\langle a^{2}\right\rangle=\left\{1, a^{2}, a^{4}, a^{6}, a^{8}, a^{10}\right\}$;

- $L=\left\langle a^{2} b, a^{3}\right\rangle=\left\{1, a^{3}, a^{6}, a^{9}, a^{2} b, a^{8} b, a^{5} b, a^{11} b\right\}$.

\subsection{A dicyclic solution of $\operatorname{HWP}(24 ; 3,4 ; 7,4)$}

Consider the four cycles of $\operatorname{Cycle}(G)$ defined as follows.

$$
\begin{aligned}
& C_{1}=\left(1, a^{3} b, a^{5}\right) \\
& C_{2}=\left(1, a^{10}, a^{7} b\right) \\
& C_{3}=\left(1, a^{4}, a^{8}\right) \\
& C_{4}=\left(1, b, a^{3} b, a\right)
\end{aligned}
$$

We note that the $\operatorname{Stab}\left(C_{3}\right)=V\left(C_{3}\right)$ while all other $C_{i}$ 's have trivial stabilizer. Thus, by Lemma 2.2, one can check that $\operatorname{Orb}\left(C_{i}\right)$ is a $\ell_{i}$-cycle decomposition of Cay $\left[G: \Omega_{i}\right]$ where $\ell_{i}$ is the length of $C_{i}$ and where the $\Omega_{i}$ 's are the symmetric subsets of $G$ listed below.

$$
\begin{aligned}
& \Omega_{1}=\left\{a^{3} b, a^{5}, a^{2} b\right\}^{ \pm 1} \\
& \Omega_{2}=\left\{a^{2}, a b, a^{5} b\right\}^{ \pm 1} \\
& \Omega_{3}=\left\{a^{4}\right\}^{ \pm 1} \\
& \Omega_{4}=\left\{b, a^{3}, a^{4} b, a\right\}^{ \pm 1}
\end{aligned}
$$

Now note that the $\Omega_{i}$ 's partition $G \backslash\left\{1, a^{6}\right\}$. Thus, by Theorem 2.3 we can say that $\mathcal{C}:=$ $\bigcup_{i=1}^{4} \operatorname{Orb}\left(C_{i}\right)$ is a $G$-regular cycle-decomposition of $K_{24}-I$. Now set $F_{i}=\operatorname{Orb}_{S_{i}}\left(C_{i}\right)$ 
where

$$
S_{i}= \begin{cases}L & \text { for } i=1,2 ; \\ G & \text { for } i=3 ; \\ K & \text { for } i=4 .\end{cases}
$$

By Remark 2.4, each $F_{i}$ is a 2-factor of $K_{24}-I$ with $\operatorname{Stab}_{G}\left(F_{i}\right)=S_{i}$, hence $\operatorname{Orb}_{G}\left(F_{i}\right)$ has length 3 or 1 or 4 according to whether $i=1,2$ or $i=3$ or $i=4$, respectively.

The cycles of $F_{i}$ are triangles or quadrangles according to whether or not $i \leq 3$. Thus, recalling that $\mathcal{C}$ is a cycle-decomposition of $K_{48}-I$, we conclude that $\mathcal{F}:=$ $\bigcup_{i=1}^{4} \operatorname{Orb}_{G}\left(F_{i}\right)$ is a $G$-regular 2-factorization of $K_{24}-I$ with 7 triangle-factors and 4 quadrangle-factors, namely a $G$-regular solution of $\operatorname{HWP}(24 ; 3,4 ; 7,4)$.

\subsection{A dicyclic solution of $\operatorname{HWP}(24 ; 3,4 ; 9,2)$}

Consider the four cycles of $\operatorname{Cycle}(G)$ defined as follows.

$$
\begin{aligned}
& C_{1}=\left(1, b, a^{6}, a^{6} b\right) \\
& C_{2}=\left(1, a^{4} b, a^{6}, a^{10} b\right) \\
& C_{3}=\left(1, a^{4}, a^{7} b\right) \\
& C_{4}=\left(1, a^{3} b, a^{8} b\right) \\
& C_{5}=\left(a^{4}, a^{7}, a^{5}\right)
\end{aligned}
$$

We note that $\operatorname{Stab}\left(C_{i}\right)=V\left(C_{i}\right)$ for $i=1,2$ while all other $C_{i}$ 's have trivial stabilizer. By Lemma 2.2, one can check that $\operatorname{Orb}\left(C_{i}\right)$ is a $\ell_{i}$-cycle decomposition of Cay $\left[G: \Omega_{i}\right]$ where $\ell_{i}$ is the length of $C_{i}$ and where the $\Omega_{i}$ 's are the symmetric subsets of $G$ listed below.

$$
\begin{aligned}
& \Omega_{1}=\{b\}^{ \pm 1} \\
& \Omega_{2}=\left\{a^{4} b\right\}^{ \pm 1} \\
& \Omega_{3}=\left\{a^{4}, a b, a^{5} b\right\}^{ \pm 1} \\
& \Omega_{4}=\left\{a^{3} b, a^{2} b, a^{5}\right\}^{ \pm 1} \\
& \Omega_{5}=\left\{a^{1}, a^{2}, a^{3}\right\}^{ \pm 1}
\end{aligned}
$$

Also here the $\Omega_{i}$ 's partition $G \backslash\left\{1, a^{6}\right\}$, hence $\mathcal{C}:=\bigcup_{i=1}^{5} \operatorname{Orb}_{G}\left(C_{i}\right)$ is a $G$-regular cycle-decomposition of $K_{24}-I$ by Theorem 2.3. Now set:

$$
\begin{array}{ll}
F_{1}=\operatorname{Orb}_{G}\left(C_{1}\right), & F_{2}=\operatorname{Orb}_{G}\left(C_{2}\right), \\
F_{3}=\operatorname{Orb}_{L}\left(C_{3}\right), & F_{4}=\operatorname{Orb}_{H}\left(C_{4}\right) \cup \operatorname{Orb}_{H}\left(C_{5}\right) .
\end{array}
$$

By Remark 2.4, each $F_{i}$ is a 2-factor of $K_{24}-I$ and we have

$$
\operatorname{Stab}_{G}\left(F_{1}\right)=\operatorname{Stab}_{G}\left(F_{2}\right)=G ; \quad \operatorname{Stab}_{G}\left(F_{3}\right)=L ; \quad \operatorname{Stab}_{G}\left(F_{4}\right)=H
$$

so that the lengths of the $G$-orbits of $F_{1}, \ldots, F_{4}$ are $1,1,3$ and 6 , respectively. The cycles of $F_{i}$ are triangles or quadrangles according to whether or not $i \geq 3$. Thus, recalling that $\mathcal{C}$ is a cycle-decomposition of $K_{48}-I$, we conclude that $\mathcal{F}:=\bigcup_{i=1}^{5} \operatorname{Orb}_{G}\left(F_{i}\right)$ is a $G$ regular 2-factorization of $K_{24}-I$ with 9 triangle-factors and 2 quadrangle-factors, namely a $G$-regular solution of $\operatorname{HWP}(24 ; 3,4 ; 9,2)$. 


\section{A special linear solution of $\operatorname{HWP}(24 ; 3,4 ; 5,6)$}

In this section $G$ will denote the 2-dimensional special linear group over $\mathbb{Z}_{3}$, usually denoted by $S L_{2}(3)$, namely the group of $2 \times 2$ matrices with elements in $\mathbb{Z}_{3}$ and determinant one. The only involution of $G$ is $2 E$ where $E$ is the identity matrix of $G$. The 2-Sylow subgroup $Q$ of $G$, isomorphic to the group of quaternions, is the following:

$$
Q=\left\{\left[\begin{array}{ll}
1 & 0 \\
0 & 1
\end{array}\right],\left[\begin{array}{ll}
2 & 0 \\
0 & 2
\end{array}\right],\left[\begin{array}{ll}
1 & 1 \\
1 & 2
\end{array}\right],\left[\begin{array}{ll}
2 & 2 \\
2 & 1
\end{array}\right],\left[\begin{array}{ll}
0 & 2 \\
1 & 0
\end{array}\right],\left[\begin{array}{ll}
0 & 1 \\
2 & 0
\end{array}\right],\left[\begin{array}{ll}
1 & 2 \\
2 & 2
\end{array}\right],\left[\begin{array}{ll}
2 & 1 \\
1 & 1
\end{array}\right]\right\}
$$

We will also need to consider the subgroup $H$ of $G$ of order 6 generated by the matrix $\left[\begin{array}{ll}0 & 1 \\ 2 & 1\end{array}\right]$. Hence we have:

$$
H=\left\{\left[\begin{array}{ll}
1 & 0 \\
0 & 1
\end{array}\right],\left[\begin{array}{ll}
0 & 1 \\
2 & 1
\end{array}\right],\left[\begin{array}{ll}
2 & 1 \\
2 & 0
\end{array}\right],\left[\begin{array}{ll}
2 & 0 \\
0 & 2
\end{array}\right],\left[\begin{array}{ll}
0 & 2 \\
1 & 2
\end{array}\right],\left[\begin{array}{ll}
1 & 2 \\
1 & 0
\end{array}\right]\right\}
$$

The use of the special linear group $G$ was crucial in [5] to get a Steiner triple system of any order $v=144 n+25$ with an automorphism group acting sharply transitively an all but one point. Here $G$ will be used to get a $G$-regular solution of the last Hamilton-Waterloo problem left open in [11].

Consider the six cycles of $\operatorname{Cycle}(G)$ defined as follows.

$$
\begin{aligned}
C_{1} & =\left(\left[\begin{array}{ll}
1 & 0 \\
0 & 1
\end{array}\right],\left[\begin{array}{ll}
2 & 0 \\
2 & 2
\end{array}\right],\left[\begin{array}{ll}
1 & 2 \\
1 & 0
\end{array}\right]\right) \\
C_{2} & =\left(\left[\begin{array}{ll}
1 & 0 \\
0 & 1
\end{array}\right],\left[\begin{array}{ll}
0 & 2 \\
1 & 2
\end{array}\right],\left[\begin{array}{ll}
2 & 1 \\
2 & 0
\end{array}\right]\right) \\
C_{3} & =\left(\left[\begin{array}{ll}
1 & 0 \\
0 & 1
\end{array}\right],\left[\begin{array}{ll}
0 & 1 \\
2 & 2
\end{array}\right],\left[\begin{array}{ll}
2 & 2 \\
1 & 0
\end{array}\right]\right) \\
C_{4} & =\left(\left[\begin{array}{ll}
1 & 0 \\
0 & 1
\end{array}\right],\left[\begin{array}{ll}
0 & 1 \\
2 & 0
\end{array}\right],\left[\begin{array}{ll}
2 & 0 \\
0 & 2
\end{array}\right],\left[\begin{array}{ll}
0 & 2 \\
1 & 0
\end{array}\right]\right) \\
C_{5} & =\left(\left[\begin{array}{ll}
1 & 0 \\
0 & 1
\end{array}\right],\left[\begin{array}{ll}
1 & 1 \\
1 & 2
\end{array}\right],\left[\begin{array}{ll}
2 & 0 \\
0 & 2
\end{array}\right],\left[\begin{array}{ll}
2 & 2 \\
2 & 1
\end{array}\right]\right) \\
C_{6} & =\left(\left[\begin{array}{ll}
1 & 0 \\
0 & 1
\end{array}\right],\left[\begin{array}{ll}
2 & 1 \\
1 & 1
\end{array}\right],\left[\begin{array}{ll}
2 & 1 \\
0 & 2
\end{array}\right],\left[\begin{array}{ll}
1 & 1 \\
0 & 1
\end{array}\right]\right)
\end{aligned}
$$

Here the stabilizer of $C_{i}$ is trivial for $i=1,6$ while it coincides with $V\left(C_{i}\right)$ for $2 \leq i \leq 5$. By Lemma 2.2, one can check that $\operatorname{Orb}\left(C_{i}\right)$ is a $\ell_{i}$-cycle decomposition of Cay $\left[G: \Omega_{i}\right]$ where $\ell_{i}$ is the length of $C_{i}$ and where the $\Omega_{i}$ 's are the symmetric subsets of $G$ listed below.

$$
\begin{aligned}
& \Omega_{1}=\left\{\left[\begin{array}{ll}
2 & 0 \\
2 & 2
\end{array}\right],\left[\begin{array}{ll}
1 & 2 \\
1 & 0
\end{array}\right],\left[\begin{array}{ll}
0 & 2 \\
1 & 1
\end{array}\right]\right\}^{ \pm 1} \\
& \Omega_{2}=\left\{\left[\begin{array}{ll}
0 & 2 \\
1 & 2
\end{array}\right]\right\}^{ \pm 1} \quad \Omega_{3}=\left\{\left[\begin{array}{ll}
0 & 1 \\
2 & 2
\end{array}\right]\right\}^{ \pm 1} \\
& \Omega_{4}=\left\{\left[\begin{array}{ll}
0 & 1 \\
2 & 0
\end{array}\right]\right\}^{ \pm 1} \quad \Omega_{5}=\left\{\left[\begin{array}{ll}
1 & 1 \\
1 & 2
\end{array}\right]\right\}^{ \pm 1} \\
& \Omega_{6}=\left\{\left[\begin{array}{ll}
2 & 1 \\
1 & 1
\end{array}\right],\left[\begin{array}{ll}
1 & 0 \\
2 & 1
\end{array}\right],\left[\begin{array}{ll}
2 & 2 \\
0 & 2
\end{array}\right],\left[\begin{array}{ll}
1 & 1 \\
0 & 1
\end{array}\right]\right\}^{ \pm 1}
\end{aligned}
$$


Once again we see that the $\Omega_{i}$ 's partition $G \backslash\{E, 2 E\}$, therefore $\mathcal{C}:=\bigcup_{i=1}^{6} \operatorname{Orb}\left(C_{i}\right)$ is a $G$-regular cycle-decomposition of $K_{24}-I$. Now set $F_{i}=\operatorname{Orb}_{S_{i}}\left(C_{i}\right)$ with

$$
S_{i}= \begin{cases}Q & \text { for } i=1 \\ G & \text { for } 2 \leq i \leq 5 \\ H & \text { for } i=6\end{cases}
$$

By Remark 2.4, each $F_{i}$ is a 2-factor of $K_{24}-I$ and we have $\operatorname{Stab}_{G}\left(F_{i}\right)=S_{i}$ so that the lengths of the $G$-orbits of $F_{1}, \ldots, F_{6}$ are $3,1,1,1,1$ and 4 , respectively.

The cycles of $F_{i}$ have length 3 or 4 according to whether or not $i \leq 3$. Thus, recalling that $\mathcal{C}$ is a cycle-decomposition of $K_{24}-I$, we conclude that $\mathcal{F}:=\bigcup_{i=1}^{6} \operatorname{Orb}_{G}\left(F_{i}\right)$ is a $G$-regular 2-factorization of $K_{24}-I$ with 5 triangle-factors and 6 quadrangle-factors, namely a $G$-regular solution of $\operatorname{HWP}(24 ; 3,4 ; 5,6)$.

\section{References}

[1] B. Alspach, P. J. Schellenberg, D. R. Stinson and D. Wagner, The Oberwolfach problem and factors of uniform odd length cycles, J. Combin. Theory Ser. A 52 (1989), 20-43, doi:10.1016/ 0097-3165(89)90059-9.

[2] S. Bonvicini and M. Buratti, Sharply vertex transitive factorizations of Cayley graphs, in preparation.

[3] S. Bonvicini, M. Buratti, G. Rinaldi and T. Traetta, Some progress on the existence of 1-rotational Steiner triple systems, Des. Codes Cryptogr. 62 (2012), 63-78, doi:10.1007/ s10623-011-9491-3.

[4] D. Bryant and V. Scharaschkin, Complete solutions to the Oberwolfach problem for an infinite set of orders, J. Combin. Theory Ser. B 99 (2009), 904-918, doi:10.1016/j.jctb.2009.03.003.

[5] M. Buratti, 1-rotational Steiner triple systems over arbitrary groups, J. Combin. Des. 9 (2001), 215-226, doi:10.1002/jcd.1008.abs.

[6] M. Buratti, Rotational $k$-cycle systems of order $v<3 k$; another proof of the existence of odd cycle systems, J. Combin. Des. 11 (2003), 433-441, doi:10.1002/jcd.10061.

[7] M. Buratti, Cycle decompositions with a sharply vertex transitive automorphism group, Le Matematiche 59 (2004), 91-105 (2006), https: / / www. dmi. unict.it/ojs/index. php/lematematiche/article/view/164.

[8] M. Buratti and P. Danziger, A cyclic solution for an infinite class of Hamilton-Waterloo problems, Graphs Combin. 32 (2016), 521-531, doi:10.1007/s00373-015-1582-x.

[9] M. Buratti and G. Rinaldi, On sharply vertex transitive 2-factorizations of the complete graph, J. Combin. Theory Ser. A 111 (2005), 245-256, doi:10.1016/j.jcta.2004.11.014.

[10] A. Burgess, P. Danziger and T. Traetta, On the Hamilton-Waterloo problem with odd orders, $J$. Combin. Des. (2016), doi:10.1002/jcd.21552.

[11] P. Danziger, G. Quattrocchi and B. Stevens, The Hamilton-Waterloo problem for cycle sizes 3 and 4, J. Combin. Des. 17 (2009), 342-352, doi:10.1002/jcd.20219.

[12] D. G. Hoffman and P. J. Schellenberg, The existence of $C_{k}$-factorizations of $K_{2 n}-F$, Discrete Math. 97 (1991), 243-250, doi:10.1016/0012-365x(91)90440-d.

[13] F. Merola and T. Traetta, Infinitely many cyclic solutions to the Hamilton-Waterloo problem with odd length cycles, Discrete Math. 339 (2016), 2267-2283, doi:10.1016/j.disc.2016.03. 026 . 
[14] U. Odabaşı and S. Özkan, The Hamilton-Waterloo problem with $C_{4}$ and $C_{m}$ factors, Discrete Math. 339 (2016), 263-269, doi:10.1016/j.disc.2015.08.013.

[15] T. Traetta, A complete solution to the two-table Oberwolfach problems, J. Combin. Theory Ser. A 120 (2013), 984-997, doi:10.1016/j.jcta.2013.01.003.

[16] L. Wang, F. Chen and H. Cao, The Hamilton-Waterloo problem for $C_{3}$-factors and $C_{n}$-factors, 2016, arXiv:1609.00453 [math.co]. 